2006 December 4

\title{
The Position of Sagittarius A*: III. Motion of the Stellar Cusp
}

\author{
M. J. Reid \\ Harvard-Smithsonian Center for Astrophysics, 60 Garden Street, Cambridge, MA 02138 \\ reid@cfa.harvard.edu \\ K. M. Menten \\ Max-Planck-Institut für Radioastronomie, Auf dem Hügel 69, D-53121 Bonn, Germany \\ kmenten@mpifr-bonn.mpg.de \\ S. Trippe, T. Ott \& R. Genzel \\ Max-Planck-Institut für extraterrestrische Physik, Postfach 1312, D-85741 Garching, \\ Germany \\ trippe@mpe.mpg.de, ott@mpe.mpg.de, genzel@mpe.mpg.de
}

\begin{abstract}
In the first two papers of this series, we determined the position of Sgr $\mathrm{A}^{*}$ on infrared images, by aligning the positions of red giant stars with positions measured at radio wavelengths for their circumstellar $\mathrm{SiO}$ masers. In this paper, we report detections of 5 new stellar SiO masers within 50" (2 pc) of Sgr A* and new and/or improved positions and proper motions of 15 stellar $\mathrm{SiO}$ masers. The current accuracies are $\approx 1$ mas in position and $\approx 0.3$ mas $\mathrm{y}^{-1}$ in proper motion. We find that the proper motion of the central stellar cluster with respect to Sgr $\mathrm{A}^{*}$ is less than $45 \mathrm{~km} \mathrm{~s}^{-1}$. One star, IRS 9, has a three-dimensional speed of $\approx 370 \mathrm{~km} \mathrm{~s}^{-1}$ at a projected distance of $0.33 \mathrm{pc}$ from Sgr A*. If IRS 9 is bound to the inner parsec, this requires an enclosed mass that exceeds current estimates of the sum of the mass of Sgr $\mathrm{A}^{*}$ and luminous stars in the stellar cusp by $\approx 0.8 \times 10^{6} \mathrm{M}_{\odot}$. Possible explanations include i) that IRS 9 is not bound to the central parsec and has "fallen" from a radius greater than $9 \mathrm{pc}$, ii) that a cluster of dark stellar remnants accounts for some of the excess mass, and/or iii) that $R_{0}$ is considerably greater than $8 \mathrm{kpc}$.
\end{abstract}


Subject headings: Galaxy: center - astrometry - masers - stars: dark matter: AGB and post AGB, variables

\section{Introduction}

Sagittarius A* (Sgr A*), the compact radio source at the center of the Galaxy (Balick \& Brown 1974), is almost surely a super-massive black hole. Infrared-bright stars orbit about the radio position of $\operatorname{Sgr~A}$ and require $\approx 3.9 \times 10^{6} \mathrm{M}_{\odot}$ within a radius of $\approx 50 \mathrm{AU}$ (Eisenhauer et al. 2005; Ghez et al. 2005). While orbiting stars move at thousands of $\mathrm{km} \mathrm{s}^{-1}$, Sgr $\mathrm{A}^{*}$ is essentially stationary at the dynamical center of the Galaxy, moving $<2 \mathrm{~km} \mathrm{~s}^{-1}$ out of the Galactic plane, Backer \& Sramek 1999; Reid, Readhead, Vermeulen \& Treuhaft 1999; Reid \& Brunthaler 2004). This indicates that the radiative source Sgr A*, which is less than 1 AU in size (Rogers et al. 2004; Krichbaum et al. 1998; Doeleman et al. 2001; Bower et al. 2004; Shen et al. 2005), contains a significant fraction $(>10 \%)$ of the gravitational mass (Reid \& Brunthaler 2004).

The Galactic Center stellar cluster contains red giant stars that are both strong radio sources (from circumstellar $\mathrm{SiO}$ maser emission) and bright infrared sources. Because these stars are visible at both radio and infrared wavelengths, one can use their radio positions, measured with respect to $\operatorname{Sgr} \mathrm{A}^{*}$, to determine accurate scale, rotation, and distortion corrections for an infrared image. This allows the highly accurate radio reference frame to be transfered to the infrared images, improving the quality of the infrared astrometry and precisely locating the position of Sgr A*. In Menten et al. (1997) and Reid et al. (2003), hereafter Papers I \& II, we developed this technique and determined the position of Sgr A* on diffraction-limited $2.2 \mu \mathrm{m}$ wavelength images of the Galactic Center with an accuracy of $\approx 15$ mas. Locating Sgr $A^{*}$ on infrared images has been important for determining its emission in the presence of confusing stellar sources and verifying that foci of stellar orbits coincide with the position of Sgr A*. This links the radiative (compact radio) source with the gravitational source.

We present new VLA observations of stellar SiO masers in the central cluster, updating their positions and, by more than doubling the observing time-span, significantly improving their proper motion determinations. We place the proper motions of infrared stars in the central cluster in a reference frame tied to $\mathrm{Sgr} \mathrm{A}^{*}$. In $\S 2$ we describe the radio measurements of the positions and proper motions of $15 \mathrm{SiO}$ maser stars, and in $\S 3$ we present the latest infrared positions and proper motions of those stars within $20^{\prime \prime}$ of Sgr A*. We use these data to transfer the infrared proper motions to a reference frame tied to Sgr A* in $\S 4$. Finally, in $\S 5$, we use the three-dimensional speeds and projected offsets of stars from Sgr $\mathrm{A}^{*}$ to 
constrain the combined mass of Sgr $\mathrm{A}^{*}$ and the central (luminous and dark) stellar cluster.

\section{Radio Observations}

Over the period 1995 to 2006 we have searched for and mapped $\mathrm{SiO}$ masers associated with late-type stars that are projected near Sgr A*. We used the NRAO1 VLBA and VLA to measure accurately the relative positions of $\mathrm{SiO}$ maser stars and $\mathrm{Sgr} \mathrm{A}^{*}$.

Red giant and super-giant stars of late-M spectral class often exhibit $\mathrm{SiO}$ masers in their extended atmospheres. These masers are strongly variable over time scales of $\sim 1 \mathrm{y}$. $\mathrm{SiO}$ maser emission emanates from a typical radius of $\sim 4 \mathrm{AU}$ (e.g., Diamond \& Kemball (2003)), which corresponds to $\sim 0.5$ mas at the assumed $8.0 \mathrm{kpc}$ distance of the Galactic Center (Reid 1993). As our measurements were made over a time-span much longer than the stellar cycle, we cannot track individual maser features, and we accept an intrinsic stellar position uncertainty of about \pm 0.5 mas owing to possible variations across the maser shell. For a late-type super-giant, the stellar radius is considerably larger than for a red giant of similar spectral class, and its $\mathrm{SiO}$ masers are found at radii roughly an order of magnitude larger. Variation of the $\mathrm{SiO}$ masers in a super-giant can considerably degrade inferred stellar position and proper motion measurements (see discussion of IRS 7 in $§ 2.1$ ).

\subsection{VLA Observations}

Our VLA observations were conducted in the A-configuration in 1995 June (reported in Paper I), 1998 May and 2000 October/November (reported in Paper II) and 2006 March (reported here). Near the Galactic Center, $\mathrm{SiO}$ masers are likely detectable over a very wide range of velocities, probably exceeding $700 \mathrm{~km} \mathrm{~s}^{-1}$. However, wide-band observations at the VLA are currently severely limited by the correlator. In order to obtain adequate spectral resolution and sensitivity, we chose to limit our velocity coverage and observe in seven $6.25 \mathrm{MHz}$ bands (each covering $\approx 40 \mathrm{~km} \mathrm{~s}^{-1}$ excluding band edges). We observed in both right and left circular polarization for each band and obtained 64 spectral channels per band, resulting in uniformly weighted spectral channel spacings of about $98 \mathrm{kHz}$ or $0.67 \mathrm{~km} \mathrm{~s}^{-1}$.

Our 2006 observations were conducted on March 5, 18, \& 19. The VLA had 24 antennas

\footnotetext{
${ }^{1}$ NRAO is a facility of the National Science Foundation operated under cooperative agreement by Associated Universities, Inc.
} 
in operation, and the synthesized beam toward Sgr A* was about $86 \times 33$ mas elongated in the north-south direction. Except for occasional calibration sources, we pointed on the position of Sgr A*, allowing detection of masers within the primary beam of a VLA antenna $\left(\approx 30^{\prime \prime} \mathrm{HWHM}\right.$ at $\left.43 \mathrm{GHz}\right)$. We observed by cycling among bands centered at LSR velocities of $-346,-111,-73,-39,-1,+40$ and $+75 \mathrm{~km} \mathrm{~s}^{-1}$; the latter six bands covered the LSR velocity range -131 to $+95 \mathrm{~km} \mathrm{~s}^{-1}$ with only two small gaps. This setup allowed for deeper integrations for the previously known, or suspected, $\mathrm{SiO}$ maser stars than would be possible for a "wide-open" search.

Initial calibration of the VLA data was done in a standard manner recommended by the Astronomical Image Processing System (AIPS) documentation. The flux density scale was based on observations of 3C 286, assuming 1.49 Jy for interferometer baselines shorter than $300 \mathrm{k} \lambda$. Amplitude and bandpass calibration was accomplished with observations of NRAO 530, which had a flux density of 2.8 Jy during the 2006 observations. The visibility data were then self-calibrated (amplitude and phase) on Sgr A* for each individual 10sec integration. This removed essentially all interferometer phase fluctuations, owing to propagation through the Earth's atmosphere, and placed the phase center at the position of Sgr $A^{*}$.

Sgr $A^{*}$ is known to vary occassionally in flux density by $\approx 10 \%$ on hourly time scales (Yusef-Zadeh et al. 2006). Were this to happen during our observations, self-calibrating on Sgr A* would introduce false gain (amplitude) variations of a similar magnitude and degrade somewhat the dynamic range of the $\mathrm{SiO}$ maser images. However, this is unlikely to shift the measured position of the masers significantly, as the positions are determined almost entirely by the phase data.

We searched for new maser stars by making very large images, covering about $\pm 50^{\prime \prime}$, or most of the primary beam of an individual VLA antenna at $43 \mathrm{GHz}$, about Sgr A* This was done by limiting the range of uv-data used and resulted in maps with a nearly circular beam of $0.15^{\prime \prime}$. Typical rms noise levels in these images were near $9 \mathrm{mJy}$, allowing $6 \sigma$ detections of 54 mJy. Five new $\mathrm{SiO}$ maser stars were discovered: SiO-14, SiO-15, SiO-16, SiO-17, and IRS $19 \mathrm{NW}$.

Once the approximate location of a maser was known, either from previous observations or from the large images, we mapped each band with up to five small sub-images centered on known or suspected masers with emission in that band. These synthesized maps typically had single spectral-channel noise levels of about $5 \mathrm{mJy}$. We always included a sub-image for Sgr $A^{*}$ at the phase center of the interferometric data. By simultaneously imaging the stellar $\mathrm{SiO}$ masers and the continuum emission from $\mathrm{Sgr} \mathrm{A}^{*}$, the strong continuum emission from $\mathrm{Sgr} \mathrm{A}^{*}$ did not degrade the detections of relatively weak $\mathrm{SiO}$ masers far from $\mathrm{Sgr} \mathrm{A}^{*}$. A 
composite spectrum from all seven observing bands for the 1998, 2000, and 2006 observations is shown in Fig. 1 .

As described in Paper II, we obtained a single position for each star at each observing epoch by 1) fitting a 2-dimensional Gaussian brightness distribution to each spectral channel with detectable $\mathrm{SiO}$ emission, 2) averaging, using variance weighting, to obtain a best stellar position and estimated uncertainty (if the reduced $\chi^{2}$ was greater than unity, we increased the position uncertainties accordingly), and 3) correcting for differential aberration, an effect of $<1$ mas for stars $<15$ " of Sgr A*.

We list the positions of the $\mathrm{SiO}$ maser stars, relative to Sgr A*, in Table 1. We include the results from the 1995 VLA observations reported in Paper I, the 1998 and 2000 VLA observations reported in Paper II, and the 2006 results reported in this paper. Since stellar $\mathrm{SiO}$ masers are variable in strength over the period of the stellar pulsation and the sensitivity of each epoch's data differed somewhat, only the stronger sources are detected at all epochs.

One of the stars, IRS 7, is a super-giant. As discussed in Paper II, it has $\mathrm{SiO}$ maser features spread over $\approx 20 \mathrm{~km} \mathrm{~s}^{-1}$ and is highly variable. We would expect its stronger $\mathrm{SiO}$ maser peaks could be spread over a region of at least 10 mas. Thus, the positions determined from the brightest $\mathrm{SiO}$ maser feature(s) in IRS 7 may not indicate the stellar position to better than about 5 mas, and we have increased the position uncertainties for IRS 7 in Table 2 to allow for this possibility.

We constructed spectra at the pixel of peak brightness for $\mathrm{SiO}$ masers detected in the 1998, 2000, and 2006 VLA observations. These spectra are displayed in Figs. 2 \& 3. Most of these $\mathrm{SiO}$ spectra are as expected for Mira variables located at the distance of the the Galactic Center: they show flux densities $\lesssim 1$ Jy covering a velocity range of 5 to $10 \mathrm{~km} \mathrm{~s}^{-1}$ and strong variability over timescales of years. Fig. 4 shows the Galactic Center region, with the positions and proper motions of the nine $\mathrm{SiO}$ maser stars that are projected within the inner 21" displayed.

\section{2. $\mathrm{SiO}$ Maser Proper Motions}

We determined stellar proper motions by fitting a variance-weighted straight line to the positions as a function of time from all of the available data compiled in Table 1. These proper motion fits are given in Table 2 and displayed graphically in Figs. 2 \& 3. The reference epoch for the proper motion solution was chosen as the variance-weighted mean epoch for each star, in order to obtain uncorrelated position and motion estimates. Since the estimated uncertainties for individual east-west and north-south positions were neither identical, nor 
exactly linearly related, we chose a single, average reference epoch for each star (instead of a separate reference epoch for the east-west and north-south directions), which resulted in slight parameter correlations.

Assuming a distance of $8.0 \mathrm{kpc}$ to the Galactic Center (Reid 1993), we convert proper motions to linear velocities and, with the radial velocities, determine the full 3-dimensional speed, $V_{\text {total }}$, of each star with respect to Sgr A*. These speeds are given in Table [3. While the speeds on the plane of the sky are directly referenced to Sgr A*, the speeds along our line-of-sight are in the LSR reference frame. Thus, our values of $V_{\text {total }}$ assume that Sgr A* has a near-zero line-of-sight speed with respect to the LSR. As no spectral lines have been detected from Sgr A*, there is no direct observational evidence supporting this assumption. However, the lack of a detectable proper motion of Sgr A* suggests that it anchors the dynamical center of the Galaxy (Reid \& Brunthaler 2004) and should be nearly at rest in the LSR frame.

\section{Infrared Observations}

Near-IR observations were obtained on the 8.2-m UT4 (Yepun) of the ESO-VLT on Cerro Paranal, Chile, using the detector system NAOS/CONICA (hereafter NACO) consisting of the adaptive optics system NAOS (Rousset et al. 2003) and the $1024 \times 1024$-pixel near-IR camera CONICA (Hartung et al. 2003). We obtained 8 data sets in $\mathrm{H}$ and $\mathrm{K}$ bands with a scale of 27 mas/pixel (large scale) covering 5 epochs (May 2002, May 2003, June 2004, May 2005, April 2006).

Each image covers a field of view (FOV) of $28 \times 28$ arcsec. During each observation the camera pointing was shifted so that the total FOV of a complete data set was between $35 \times 35$ and $40 \times 40$ arcsec, centered on Sgr A*. In all cases the spatial resolution was (nearly) diffraction limited, leading to a typical FWHM of $\sim 60$ mas. Typical limiting magnitudes were $18^{\text {th }} \mathrm{mag}$ at $\mathrm{K}$ band and $20^{\text {th }} \mathrm{mag}$ at $\mathrm{H}$ band. All images were sky-subtracted, badpixel removed and flat-field corrected. In order to obtain the best signal-to-noise ratios and maximum FOV coverages in single maps, we combined all good-quality images belonging to the same data set to mosaics after correcting for instrumental geometric distortion. Details of the distortion correction will be given in Trippe et al. (in preparation).

In order to establish an astrometric near-IR reference frame, we selected one high-quality mosaic (May 2005), extracted image positions for all detected stars and transformed them into astrometric coordinates using the positions of all 9 maser stars in the FOV interpolated to the epoch of the image. To compare positions among images of different epochs, we chose 
an ensemble of $\approx 600$ "well-behaved" stars (i.e., stars that are well-separated from neighbors and are bright but not saturated) in the "master mosaic" and computed in each image all stellar positions relative to this ensemble. Due to varying FOVs, the number of stars usable for a given mosaic varied between about 400 to 600 .

In each image, positions were extracted by fitting stars with 2-dimensional elliptical Gaussian brightness distributions. Although over the entire FOV significant departures from isoplanicity occur, this effect elongates the stellar PSFs symmetrically and does not affect significantly the centroids of Gaussian-fitted positions. Proper motions were computed by fitting the positions versus times with straight lines. To assure statistically "clean" errors for the proper motions, outlier rejection and error rescaling were applied where possible. This led to typical position accuracies of $\approx 1$ mas and typical proper motion accuracies of $\approx 0.3$ mas $^{-1}$. Unfortunately, this accuracy was not achieved for all maser stars, as some are very bright stars and are affected by detector non-linearity/saturation in some images; also the star most distant from Sgr A* (IRS19NW) was observed only in the last two epochs. Thus, some of the maser stars have errors larger than typical.

\section{Radio \& Infrared Frame Alignments}

The proper motions of stars in the Galactic Center cluster, measured from infrared images, are relative motions only. One can add an arbitrary constant vector to all of the stellar proper motions without violating observational constraints. Until now, the "zero points" of the motions have been determined by assuming isotropy and removing the average motion of the entire sample. Since the radio proper motions are inherently in a reference frame tied directly to $\mathrm{Sgr} \mathrm{A}^{*}$, one can use any one of the $\mathrm{SiO}$ stars, or the mean motion of a group of them, to place the infrared proper motions in Sgr $\mathrm{A}^{*}$ 's frame.

We have measured radio positions and proper motions, relative to Sgr $\mathrm{A}^{*}$, for the nine bright $\mathrm{SiO}$ maser stars that appear on the NACO images (ie, within $\approx 20^{\prime \prime}$ of Sgr $\mathrm{A}^{*}$ ). The position and proper motion accuracies typically are $\sim 1$ mas and $\sim 0.3$ mas $^{-1}$, respectively. This allows us to align the radio and infrared frames, both in position and in proper motion. Thus, not only can the location of Sgr A* can be accurately determined on infrared images, but also stellar proper motions from infrared data can be referenced directly to Sgr A*, without assumptions of isotropy or homogeneity of the stellar motions.

The radio and infrared proper motions measured for the nine stars are listed in Table 4 . The nine stars have weighted mean differences (and standard errors of the means) of $+0.66 \pm$

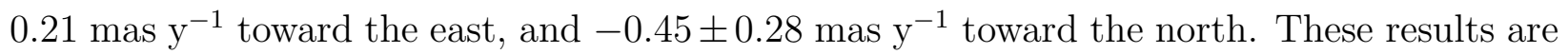


qualitatively similar to those published in Paper II. Quantitatively, the differences between the IR motions of Paper II and this paper for some stars (notably IRS 12N) are greater than expected based on the quoted uncertainties. Since the IR motions in Paper II were based on 2 epochs only, which did not allow for an internal check on the formal motion uncertainties, we believe those uncertainties were somewhat optimistic.

Currently only one star, IRS 7, has a significant discrepancy between the radio and infrared motions in the both coordinates. This is the only super-giant star in the sample and, owing to its extreme brightness, the infrared measurements are compromised by detector saturation. Additionally, the radio measurements are subject to significant uncertainty from the large $\mathrm{SiO}$ maser shell size. After removing IRS 7, the weighted mean differences between the radio and IR motions change only slightly and become $+0.63 \pm 0.21$ mas $^{-1}$ toward the east, and $-0.32 \pm 0.18$ mas $^{-1}$ toward the north.

When comparing how well the IR frame matches the radio frame, we need to consider the statistical uncertainty of the average IR motion, which has been removed. For most epochs, the average motion is based on $\approx 400$ stars, each of which has a typical motion of $\approx 100 \mathrm{~km} \mathrm{~s}^{-1}$. Thus, the mean IR motion should have an uncertainty of roughly $100 \mathrm{~km} \mathrm{~s}^{-1} / \sqrt{400} \approx 5 \mathrm{~km} \mathrm{~s}^{-1}$. Adopting the result with IRS 7 removed, converting to linear speeds for a distance of $8.0 \mathrm{kpc}$ to the Galactic Center (Reid 1993), and adding in quadrature $\mathrm{a} \approx 5 \mathrm{~km} \mathrm{~s}^{-1}$ uncertainty for the mean IR motion removed from each coordinate, implies that the infrared stellar cluster moves $+24 \pm 9 \mathrm{~km} \mathrm{~s}^{-1}$ toward the east, and $-12 \pm 9 \mathrm{~km} \mathrm{~s}^{-1}$ toward the north, with respect to Sgr A*. The northward component motion does not deviate significantly from zero; the eastward component formally presents a $2.7 \sigma$ significance. Combining these components in quadrature formally yields a speed difference of $27 \pm 9 \mathrm{~km} \mathrm{~s}^{-1}$. However, at this time, we do not consider that we have firmly detected motion of the stellar cluster, and we adopt a $2 \sigma$ upper limit of $45 \mathrm{~km} \mathrm{~s}^{-1}$ for the proper motion of the stellar cusp with respect to $\mathrm{Sgr} \mathrm{A}^{*}$.

\section{Enclosed Mass versus Radius from Sgr A*}

Estimates of the enclosed mass versus projected radius from Sgr A*, based on infrared stellar motions, rely on relative motions not tied directly to Sgr A*. Since, the 3-dimensional motions of the $\mathrm{SiO}$ masers in this paper are both very accurate and directly tied to $\mathrm{Sgr} \mathrm{A}^{*}$, they provide valuable information on the enclosed mass within projected radii of 0.2 to $2 \mathrm{pc}$ of $\operatorname{Sgr} \mathrm{A}^{*}$.

In Paper II, we derived a lower limit to the enclosed mass at the projected radius of each 
star, assuming the stellar motions reflect gravitational orbits dominated by a central point mass. Given the 3-dimensional speed, $V_{t o t a l}$, and projected distance from Sgr $\mathrm{A}^{*}, r_{\text {proj }}$, for each star, we obtained a strict lower limit to the mass enclosed, $M_{\text {encl }}$, within the true radius, $r$, of that star from Sgr A*. For a given enclosed mass, semi-major axis and eccentricity $(e)$, the greatest orbital speed occurs at pericenter for $e \approx 1$. Since the projected pericenter distance cannot exceed the true distance, we obtained

$$
M_{\text {encl }} \geq \frac{V_{t o t a l}^{2} r_{\text {proj }}}{2 G}
$$

Note that this enclosed mass limit is a factor of two lower than would be obtained for a circular orbit. This lower limit approaches an equality only when three criteria are met: 1) $r_{\text {proj }} \approx r, 2$ ) the star is near pericenter, and 3) it has an eccentricity near unity. The a priori chance of any star satisfying all three of these criteria is small, especially since a star in a highly eccentric orbit spends very little time near pericenter. Thus, Eq. (1) provides a very conservative limit on enclosed mass.

We evaluate the lower limit to $M_{\text {encl }}$ using Eq. 1 by adopting conservatively the smallest total velocity allowed by measurement uncertainties, i.e., by subtracting $2 \sigma$ from $V_{\text {total }}$ in Table 3 before calculating a mass limit. The mass limits, given in Table 3, are mostly consistent with the enclosed mass versus projected distance from Sgr A* given by Genzel, Eckart, Ott, \& Eisenhauer (1997) and Ghez, Klein. Morris \& Becklin (1998). For many of the stars, the lower mass limits are well below the estimated enclosed mass curves, as expected given the very conservative nature of the calculated limits.

Our most significant lower mass limit is from IRS 9. In Paper II, we arrived at a limit $>4.5 \times 10^{6} \mathrm{M}_{\odot}$, which exceeded the then favored model of a $2.6 \times 10^{6} \mathrm{M}_{\odot}$ black hole (Genzel, Eckart, Ott, \& Eisenhauer 1997; Ghez, Klein. Morris \& Becklin 1998), plus a $0.4 \times 10^{6} \mathrm{M}_{\odot}$ contribution from the central stellar cluster (Genzel et al. 2003), by about $50 \%$. With our improved proper motions, we now find a more stringent limit of $>5.1 \times 10^{6} \mathrm{M}_{\odot}$ at a projected radius of $0.33 \mathrm{pc}$ from Sgr A*.

Fig. 5 displays our enclosed mass versus radius constraint based on the 3-dimension motion of IRS 9, along with other constraints in the recent literature. The current best estimate for the mass of the $\mathrm{SMBH}\left(\mathrm{Sgr} \mathrm{A}^{*}\right)$ is $(3.9 \pm 0.2) \times 10^{6} \mathrm{M}_{\odot}$, for the distance to the Galactic Center, $R_{0}$, of $8.0 \mathrm{kpc}$. This mass estimate comes from an unweighted average of the results of Eisenhauer et al. (2005) and Ghez et al. (2005), based on stellar orbit determinations. Adding in a $0.4 \times 10^{6} \mathrm{M}_{\odot}$ contribution from the central stellar cluster, based on a density profile of $1.2 \times 10^{6}(r / 0.39 \mathrm{pc})^{-1.4} \mathrm{M}_{\odot} \mathrm{pc}^{-3}$ by Genzel et al. (2003), yields $4.3 \times 10^{6} \mathrm{M}_{\odot}$, still leaving a discrepancy of $0.8 \times 10^{6} \mathrm{M}_{\odot}$, for $R_{0}=8 \mathrm{kpc}$. Formally, this is about a $3 \sigma$ discrepancy, assuming an uncertainty of $\pm 0.2 \times 10^{6} \mathrm{M}_{\odot}$ in the mass estimate of 
Sgr $A^{*}$ and an estimated $\pm 30 \%$ uncertainty in the mass of the stellar cusp.

Since we do not know the line-of-sight distance of IRS 9 from Sgr A*, one might be tempted to argue that $r \approx 2 r_{\text {proj }}$ and the star is simply sensing an enclosed mass of $1.0 \times$ $10^{6} \mathrm{M}_{\odot}$ from the central stellar cluster at that radius. However, the mass limit derived from Eq. (1) scales as $r$ and would be approximately $10^{7} \mathrm{M}_{\odot}$ for $r=2 r_{\text {proj }}=0.66 \mathrm{pc}$. Thus, the mass discrepancy only increases for $r>r_{\text {proj }}$, as shown by the slanted line in Fig. 5 (but see $\S 5.2)$.

How can the lower limit to the enclosed mass provided by IRS 9 be explained? We now discuss some possibilities.

\subsection{Dark Matter in the Central Stellar Cluster}

One could explain the motion of IRS 9, were the central stellar cluster to contain dark matter (in addition to Sgr $\mathrm{A}^{*}$ ) whose mass exceeds $0.8 \times 10^{6} \mathrm{M}_{\odot}$ within $r=0.33$ pc. Morris (1993) estimates that $\sim 10^{6} \mathrm{M}_{\odot}$ of "dark" stellar remnants (eg, white dwarfs, neutron stars, black holes) could have accumulated in the inner few tenths of a parsec of the Galaxy. Mouawad et al. (2005) show that, with data available at the time, the orbital fit of star S2 allows for (but does not require) $0.2 \times 10^{6} \mathrm{M}_{\odot}$ of dark matter distributed within 0.001 pc of Sgr A*. Should such a dark component exist and extend to greater radii, it might explain some of the IRS 9 mass discrepancy. However, other estimates of the total mass in black holes in the central few tenths of a pc do not exceed $\sim 0.2 \times 10^{6} \mathrm{M}_{\odot}$ (Miralda-Escudé \& Gould 2000; Freitag. Amaro-Seoane \& Kalogera 2006). Given these estimates and the evidence from other enclosed mass indicators that do not support $\sim 10^{6} \mathrm{M}_{\odot}$ of dark matter within $\approx 1$ pc of Sgr A* (Genzel et al. 2000), it seems unlikely that a dark component could explain more than a modest fraction of the IRS 9 mass discrepancy.

\subsection{IRS 9 not bound to the central parsec}

A critical assumption for calculating the minimum enclosed mass using IRS 9's space velocity (Eq. 1) is that it is in a bound orbit dominated by a central point mass. If IRS 9 is in a highly eccentric orbit with a semimajor axis greater than a few parsecs, this assumption

can break down. In such a case, the star's space velocity could exceed the "local" escape velocity, based on the mass enclosed at its current radius, but still be bound at a larger radius. For example, a star could be bound by mass within $\approx 10$ pc of Sgr A* but observed plunging into the inner few tenths of a parsec at a speed that makes it appear unbound. 
This could happen via gravitational scattering starting either at small radii and increasing orbital energy or at large radii and removing angular momentum. van Langevelde et al. (1992) suggest a similar explanation for three $\mathrm{OH} / \mathrm{IR}$ stars with large radial velocities; these stars are seen projected tens of parsecs from Sgr $\mathrm{A}^{*}$ and would require semimajor axes of a few kpc.

Consider a Galactic Center star with a large semimajor axis and little angular momentum $(e \approx 1)$, so that it essentially "falls" toward the Center. We derive the infall velocity for a star that starts "falling" at a radius, $r_{\max }$ and reaches a radius of $r_{0}$. Assume a central point mass, $M_{B H}$, plus an extended component with density, $\rho(r)$. The kinetic energy gained by a star falling from $r$ to $r_{0}$ is equal to the difference in gravitational potential energy at those radii. Gravitational potential energy per unit mass, $U_{m}$, for a spherically symmetric mass distribution has the properties that for mass interior to $r$

$$
U_{m}=-\frac{G}{r} \int_{0}^{r} \rho(r) 4 \pi r^{2} d r
$$

and for mass exterior to $r$

$$
U_{m}=-G \int_{r}^{r_{\max }} \frac{\rho(r)}{r} 4 \pi r^{2} d r
$$

(For spherically symmetric systems, interior mass acts identically as a point mass equal to the enclosed mass located at the center of the distribution, while exterior mass results in zero gravitational force and a constant gravitational potential dependent on its radial position, but independent of the position of a "test" mass.)

Following Genzel et al. (2003), $\rho(r)=\rho_{0}\left(r / r_{0}\right)^{\alpha}$, where $\rho_{0}=1.2 \times 10^{6} \mathrm{M}_{\odot} \mathrm{pc}^{-3}$, $r_{0}=0.39 \mathrm{pc}$, and $\alpha=-2.0$ for $r \geq r_{0}$ pc and $\alpha \approx-1.4$ for $r<r_{0}$ pc. This leads to an enclosed stellar mass within $r_{0}$ of $M_{0} / 1.6$, where $M_{0}=4 \pi \rho_{0} r_{0}^{3}=0.9 \times 10^{6} \mathrm{M}_{\odot}$, plus a contribution of $M_{B H}$ from Sgr $\mathrm{A}^{*}$. Adding the contributions to the potential from different mass components for a star at radius $r$ (for $r \geq r_{0}$ ) gives

$$
U_{m}=-\frac{G}{r} M_{B H}-\frac{G}{r} \int_{0}^{r_{0}} \rho(r) 4 \pi r^{2} d r-\frac{G}{r} \int_{r_{0}}^{r} \rho(r) 4 \pi r^{2} d r-G \int_{r}^{r_{\max }} \frac{\rho(r)}{r} 4 \pi r^{2} d r
$$

The first three terms on the right hand side of Eq. (2) sum the effects of the mass components interior to $r$, and the fourth term is the contribution from the mass exterior to $r$. Evaluating Eq. (2) we find

$$
U_{m}=-\frac{G}{r} M_{B H}-\frac{G}{r} \frac{M_{0}}{1.6}-\frac{G}{r} M_{0}\left(\frac{r}{r_{0}}-1\right)-\frac{G}{r_{0}} M_{0}\left(\ln \left(r_{\max } / r_{0}\right)-\ln \left(r / r_{0}\right)\right) .
$$


For a star "falling" from $r_{\max }$ to $r_{0}$, the kinetic energy per unit mass gained is equal to the difference in potential energy per unit mass. From Eq. (3), we find

$$
\frac{1}{2} v^{2}=U_{m}\left(r_{\max }\right)-U_{m}\left(r_{0}\right)
$$

where

$$
U_{m}\left(r_{\max }\right)=-\frac{G}{r_{\max }}\left(M_{B H}+M_{0}\left(\frac{r_{\max }}{r_{0}}-0.38\right)\right)
$$

and

$$
U_{m}\left(r_{0}\right)=-\frac{G}{r_{0}}\left(M_{B H}+M_{0}\left(0.62+\ln \left(r_{\max } / r_{0}\right)\right)\right)
$$

Evaluating Eq. (4) for $r_{0}=0.39 \mathrm{pc}$, which is a reasonable value for the 3 -dimensional radius of IRS 9 , gives $v>370 \mathrm{~km} \mathrm{~s}^{-1}$ for an initial radius $r_{\max }>9 \mathrm{pc}$. Thus, if IRS 9 is in a highly eccentric orbit that takes it out to a radius of $>9 \mathrm{pc}$, it could achieve its very high observed 3-D velocity without violating the enclosed masses estimated by other methods.

A priori it might seem very unlikely that even one of 15 stars with detectable $\mathrm{SiO}$ masers would have such an orbit and be observed near closest approach to Sgr A* (where it spends little time). However, it is beyond the scope of this paper to evaluate the likelihood, especially with the limited statistics available at this time.

\subsection{The distance to the Galactic Center $\left(R_{0}\right)$ exceeds 9 kpc}

Were the mass of Sgr $\mathrm{A}^{*}>4.7 \times 10^{6} \mathrm{M}_{\odot}$, no mass discrepancy would exist. The best current mass estimates are based on fitting orbits for many stars and should be robust. However, the greatest uncertainty in the mass of Sgr A* comes its strong dependence on the adopted value of $R_{0}=8.0 \mathrm{kpc}$ for the distance to the Galactic Center. Eisenhauer et al. (2005) derive central masses from orbit fitting of $4.06 \times 10^{6} \mathrm{M}_{\odot}$ when adopting $R_{0}=8.0 \mathrm{kpc}$ and $3.61 \times 10^{6} \mathrm{M}_{\odot}$ for a best fit $R_{0}=7.62 \mathrm{kpc}$. These values suggest an enclosed mass $M_{\text {encl }} \propto R_{0}^{2.4}$. Our mass limit based on IRS 9's 3-D motion would also increase with $R_{0}$, but more weakly. Since the LSR velocity is the dominant component in the 3-dimensional motion for IRS 9 (and is not dependent on $R_{0}$ ), our minimum mass estimate (Eq. 1) scales approximately as $M_{\text {encl }} \propto R_{0}^{1.3}$, mostly through $r_{\text {proj }}$. Allowing $R_{0}$ to increase to about $9 \mathrm{kpc}$ removes the mass discrepancy. However, such a large value for $R_{0}$ seems very unlikely (Reid) 1993; Eisenhauer et al. 2005). 


\subsection{Non-zero $V_{\mathrm{LSR}}$ for $\operatorname{Sgr} \mathrm{A}^{*}$}

Were Sgr A* moving toward the Sun along the line-of-sight with a speed $>30 \mathrm{~km} \mathrm{~s}^{-1}$, this would lower $V_{\text {total }}$ and, hence, the $M_{\text {encl }}$ limit to $>4.4 \times 10^{6}$. While it seems very unlikely that a super-massive object would have such a large motion (Reid et al. 2003), we now consider this possibility. One method to approach this problem is to average the velocities of large samples of stars very close to Sgr A*, assuming that this average would apply to Sgr A*.

Our sample of $\mathrm{SiO}$ maser stars, which should be nearly complete in the LSR velocity range -131 to $+95 \mathrm{~km} \mathrm{~s}^{-1}$, does not show any obvious bias. Infrared observations of CO band-head velocities from late-type stars in the central parsecs yield average LSR velocities that are not statistically different from zero. For example, the integrated CO-band head velocities (within a 20" diameter aperture) of McGinn et al. (1989) indicate positive (negative) velocities at positive (negative) Galactic longitude, consistent with the direction of Galactic rotation, and a value of $-10 \pm 25 \mathrm{~km} \mathrm{~s}^{-1}$ at the position of Sgr A*. (However, these authors find possibly significant stellar velocities of $-47 \pm 8 \mathrm{~km} \mathrm{~s}^{-1}$ for four pointing offsets perpendicular to the Galactic plane.) Individual stellar velocities compiled by Rieke \& Rieke (1988) of 54 stars projected within $\approx 6$ pc of Sgr A* have a mean velocity of $-20 \pm 11 \mathrm{~km} \mathrm{~s}^{-1}$. Alternatively, Winnberg et al. (1985) and Sjouwerman et al. (1998) measured velocities of $\mathrm{OH}$ masers for 33 and $229 \mathrm{OH} / \mathrm{IR}$ stars, respectively, within about $\approx 40 \mathrm{pc}$ of Sgr $\mathrm{A}^{*}$, which yield average velocities of $+7 \pm 11$ and $+4 \pm 5 \mathrm{~km} \mathrm{~s}^{-1}$. Overall, it appears that radial velocities of stars near Sgr A* suggest an average LSR velocity near zero, within $\approx 20 \mathrm{~km} \mathrm{~s}^{-1}$.

\subsection{IRS 9 is (or was) in a binary}

Were IRS 9 in a tight, massive binary, perhaps a significant portion of its space velocity might be contributed by internal orbital motion, possibly reducing its speed with respect to Sgr A*. However, we have observed IRS 9 for about 8 years and see no changes in its radial or proper motion velocity components. The spectra of IRS 9 shown in Fig. 2 are characteristic of Mira variables, which show variable emission over a range of 5 to $10 \mathrm{~km} \mathrm{~s}^{-1}$ about the stellar velocity. We estimate that the stellar radial velocity of IRS 9 has changed by less than $2 \mathrm{~km} \mathrm{~s}^{-1}$ over 8 years. Also, the proper motion components are well-fit by constant velocities, with $2 \sigma$ upper limits to accelerations of 0.4 and 0.6 mas $\mathrm{y}^{-2}$ (15 and $23 \mathrm{~km} \mathrm{~s}^{-1} \mathrm{y}^{-1}$ at $8.0 \mathrm{kpc}$ ) for the eastward and northward components, respectively.

The observed changes in radial velocity (or proper motion components) would have 
different characteristics depending on the relative values of the time span of the observations, $\Delta t$, and the orbital period, $P$. For $\Delta t \geq P / 4$, we would have sampled large changes in the orbital mean anomaly and hence would have seen quasi-random changes of magnitude equal to the orbital speed. Our limit of $<2 \mathrm{~km} \mathrm{~s}^{-1}$ change in the radial velocity over $8 \mathrm{y}$, would place a limit of $\approx 2 \mathrm{~km} \mathrm{~s}^{-1}$ for the radial component of any orbital velocity. Alternatively, if $\Delta t<P / 4$, then we could be sampling only a small portion of an orbit and detecting a velocity change might be difficult. However, setting $\Delta t=8$ y requires $P>32$ y. Since any bright companion for IRS 9 would have been observed, we adopt companion mass $m$ of $\leq 10 \mathrm{M}_{\odot}$. This would even allow for most black hole companions. For $m<10 \mathrm{M}_{\odot}$ and $P>32 \mathrm{y}$, we find an upper limit for an orbital speed of $<20 \mathrm{~km} \mathrm{~s}^{-1}$. Thus, a binary orbital contribution to the observed space velocity of IRS 9 could not exceed $\approx 20 \mathrm{~km} \mathrm{~s}^{-1}$ and likely would be considerably less. Thus, it is highly unlikely that the extreme velocity of IRS 9 could be explained as owing to a binary orbit.

Could IRS 9 have been in a binary system and come unbound (or bound at a much larger radius as discussed in \$5.2) after a close encounter with Sgr A*? A small number of "hypervelocity" stars are thought to have been ejected from the Galactic Center in this manner (Hills 1988; Yu \& Tremaine 2003; Brown et al. 2005). However, these are estimated to be very rare events $\left(<1\right.$ in $\left.10^{5} \mathrm{y}\right)$ and we are statistically unlikely to be witnessing a newly created hyper-velocity star so close to Sgr A*. All hyper-velocity stars discovered to date are early-type main-sequence stars; they are found in the outer Galaxy and are moving at speeds of $\sim 1000 \mathrm{~km} \mathrm{~s}^{-1}$, even after climbing out of the gravitational potential of the inner Galaxy. Main sequence stars can survive the strong tidal forces experienced during close encounters with Sgr A*. However, IRS 9 is an AGB star and, thus, is a very extended $(\approx 300$ solar radii) and low surface-gravity object. It is unclear if such a star could survive the ejection event, without losing its extended atmosphere.

\section{Conclusions}

We have now measured the radio positions and proper motions of 15 late type stars with $\mathrm{SiO}$ maser emission in the Galactic Center stellar cluster. All but two of these stars have been detected at three or more epochs and have measurement accuracies of $\approx 1$ mas in position and $\approx 0.3$ mas $^{-1}$ in proper motion. Nine of these stars have multi-epoch measurements of proper motions at infrared wavelengths. A comparison of the radio motions, which are relative to Sgr $A^{*}$, with the infrared motions indicates that the stellar cusp moves with Sgr A* to within $45 \mathrm{~km} \mathrm{~s}^{-1}$.

The three-dimensional speeds and projected distances of individual stars from Sgr A* 
yield lower limits to the enclosed mass. The enclosed mass limit for one star, IRS 9, exceeds current estimates of the combined mass of Sgr A* and the luminous stars in the cusp within the central parsec. This result is puzzling, but might be explained, for example, by a combination of i) a population of dark stellar remnants in the central parsec, ii) IRS 9 being on a plunging "orbit" with a semimajor axis $\gg 1 \mathrm{pc}$, and/or iii) a value of $R_{0}>8 \mathrm{kpc}$.

We thank S. Gillessen for comments on the paper, and A. Loeb and R. O'Leary for discussions on the extreme motion of IRS 9.

\section{REFERENCES}

Alexander, T. \& Loeb, A. 2001, ApJ, 551, 223

Backer, D. C. \& Sramek, R. A. 1999, ApJ, 524, 805

Balick, B. \& Brown, R. L. 1974, ApJ, 194, 265

Beloborodov, A. M., Levin, Y., Eisenhauer, F., Genzel, R., Paumard, T., Gillessen, S. \& Ott, T. 2006, astro-ph/0601273

Bower, G. C. \& Backer, D. C. 1998, ApJ, 496, L97

Bower, G. C., Falcke, H., Herrnstein, R. M., Zhao, J.-H., Goss, W. M. \& Backer, D. C. 2004, Science, 304, 704

Brown, W. R., Geller, M. J., Kenyon, S. J. \& Kurtz, M. J. 2005, ApJ, 622, L33

Chatterjee, P., Hernquist, L., \& Loeb, A. 2002, ApJ, 572, 371

Diamond, P. J. \& Kemball, A. J. 2003, AJ, 599, 1372

Doeleman, S. S. et al. 2001, AJ, 121, 2610

Eckart, A. \& Genzel, R. 1996, Nature, 383, 415

Eckart, A. \& Genzel, R. 1997, MNRAS, 284, 576

Eckart, A., Genzel, R., Ott, T., \& Schödel, R. 2002, MNRAS, 331, 917

Eisenhauer et al.2005, ApJ, 628, 246

Fomalont, E. B., Goss, W. M., Lyne, A. G., Manchester, R. N., \& Justtanont, K. 1992, MNRAS, 258, 497 
Freitag, M., Amaro-Seoane, P. \& Kalogera, V. 2006, astro-ph/0603280

Genzel, R., Eckart, A., Ott, T., \& Eisenhauer, F. 1997, MNRAS, 291, 219

Genzel, R., Pichon, C., Eckart, A., Gerhard, O. E., \& Ott, T. 2000, MNRAS, 317, 348

Genzel, R. et al. 2003, ApJ, 594, 812

Ghez, A. M., Klein, B. L., Morris, M., \& Becklin, E. E. 1998, ApJ, 509, 678

Ghez, A. M., Morris, M., Becklin, E. E., Tanner, A., \& Kremenek, T. 2000, Nature, 407, 349

Ghez, A. M. et al. 2005, ApJ, 620, 744

Hartung, M. et al.2003, SPIE, 4841, 425

Hills, J. G. 1988, Nature, 331, 687

Hornstein, S. D., Ghez, A. M., Tanner, A., Morris, M., Becklin, E. E., \& Wizinowich, P. 2002, ApJ, 577, L9

Krichbaum, T. P. et al.1998, A\&A, 335, L106

Lada, C. J. \& Reid, M. J. 1978, ApJ, 219, 95

van Langevelde, H. J., Brown, A. G. A., Lindqvist, M., Habing, H. J. \& de Zeeuw, P. T. 1992, A\&AS, 261, L17

McGinn, M. T., Sellgren, K., Becklin, E. E. \& Hall, D. N. B. 1989, ApJ, 338, 824

Menten, K. M., Reid, M. J., Eckart, A.\& Genzel, R. 1997, ApJ, 475, L111 (Paper I)

Miralda-Escudé, J. \& Gould, A. 2000, ApJ, 545, 847

Morris, M. 1993, ApJ, 408, 496

Mouawad, N., Eckart, A., Pfalzner, S., Schödel, R., Moultaka, J. \& Spurzem, R. 2005, Astronomische Nachrichten, 326 (Issue 2), 83

Reid, M. J. 1993, ARA\&A, 31, 345

Reid, M. J. \& Brunthaler, A. 2004, ApJ, 616, 872

Reid, M. J. \& Menten, K. M. 1997, ApJ, 476, 327 
Reid, M. J. Menten, K. M., Genzel, R. Ott, T. \& Schödel, R., 2003, ApJ, 587, 208 (Paper II)

Reid, M. J., Readhead, A. C. S., Vermeulen, R. C. \& Treuhaft, R. N. 1999, ApJ, 524, 816

Rieke, G. H. \& Rieke, M. J. 1988, ApJ, 330, L33

Rogers, A. E. E. et al. 2004, ApJ, 434, L59

Rousset, G. et al.2003, SPIE, 4839, 140

Schödel, R. et al. 2002, Nature, 419, 694

Shen, Z.-Q., Lo, K. Y., Liang, M.-C., Ho, P. T. P. \& Zhao, J.-H. 2005, Nature, 438, 62

Sjouwerman, L. O., van Langevelde, H. J., Winnberg, A., \& Habing, H. J. 1998, A\&AS, 128, 35

Trippe, S. et al. in preparation

Wardle, M. \& Yusef-Zadeh, F. 1992, ApJ, 387, L65

Winnberg, A, Baud, B., Matthews, H. E., Habing, H. J \& Olnon, F. M. 1985, ApJ, 291, L45

Yu, Q. \& Tremaine, S. 2003, ApJ, 599, 1129

Yusef-Zadeh, F., Roberts, D., Wardle, M., Heinke, C. O. \& Bower, G. C. 2006, ApJ, 650, 189. 


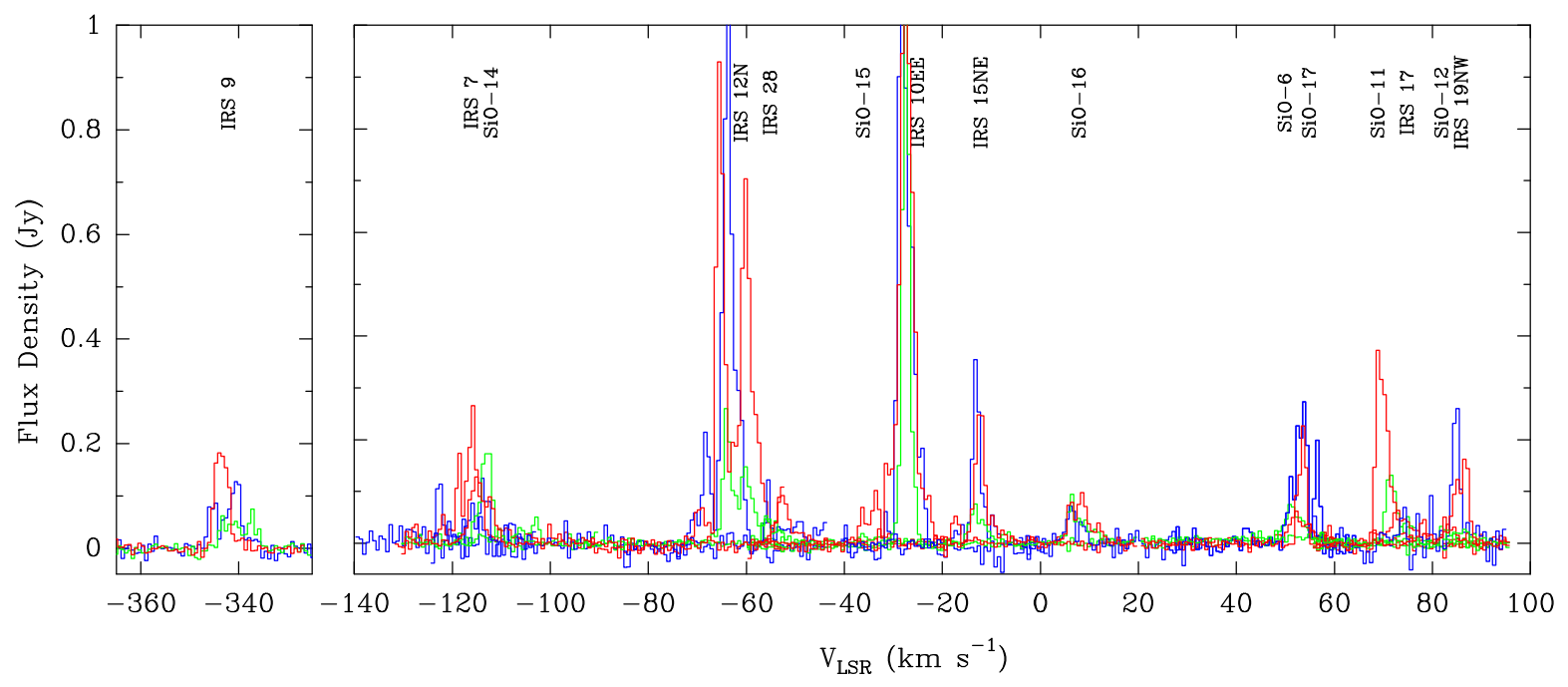

Fig. 1.- Composite spectrum of stellar $\mathrm{SiO}$ masers detected with the VLA in 1998 (blue), 2000 (green) and 2006 (red) observations. Stars are identified at the top of the spectrum at their approximate stellar velocities. 

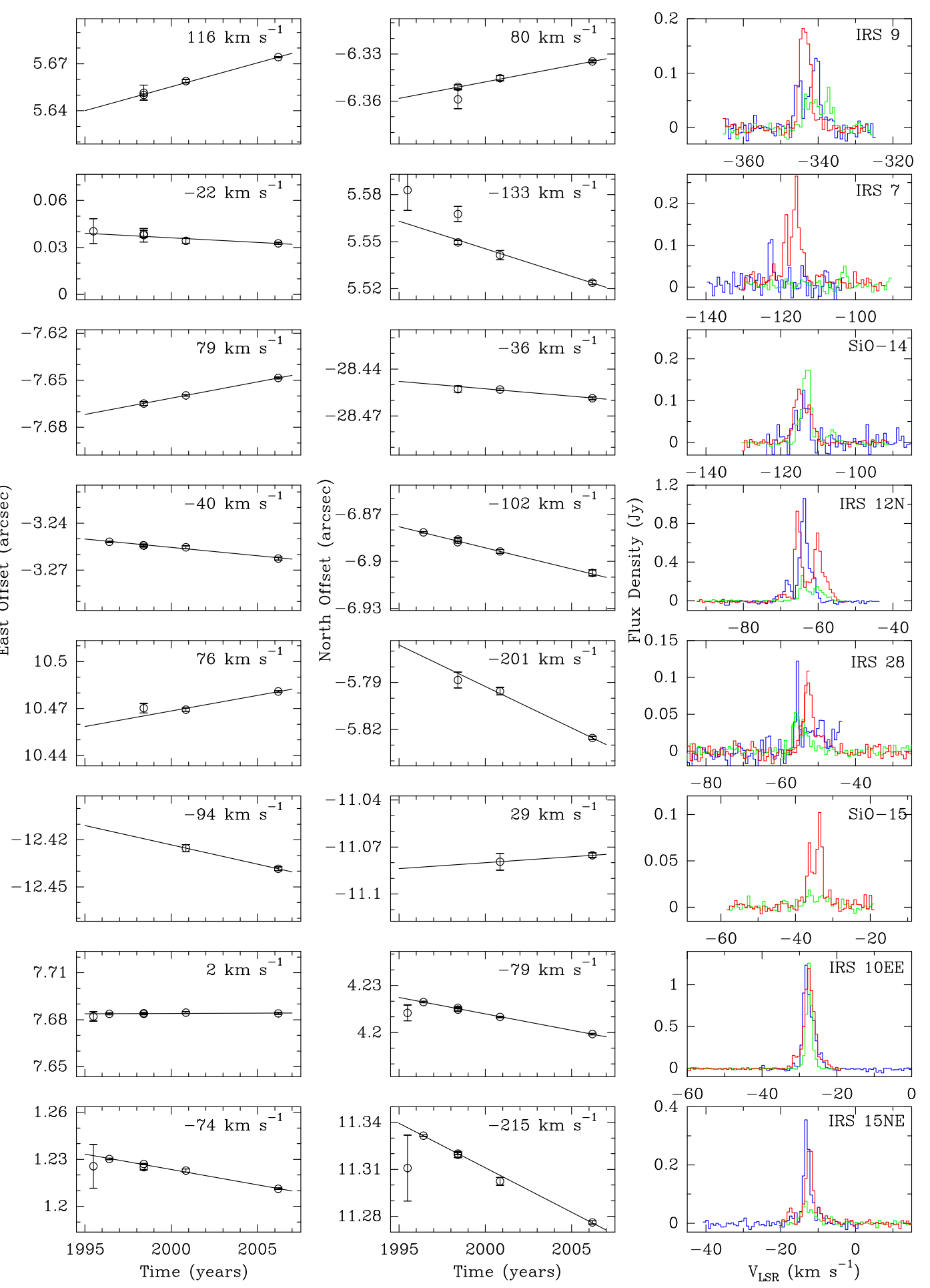

Fig. 2.- Eastward (left panels) and northward (middle panels) position offsets from Sgr A* versus time for the eight $\mathrm{SiO}$ maser stars with negative LSR velocities. Solid lines are variance-weighted best-fit proper motions. The linear speed is indicated in each frame, assuming a distance of $8.0 \mathrm{kpc}$. Spectra from 1998 (blue), 2000 (green) and 2006 (red) observations are also shown (right panels). Star names are indicated in the upper right corner of the right panels. 

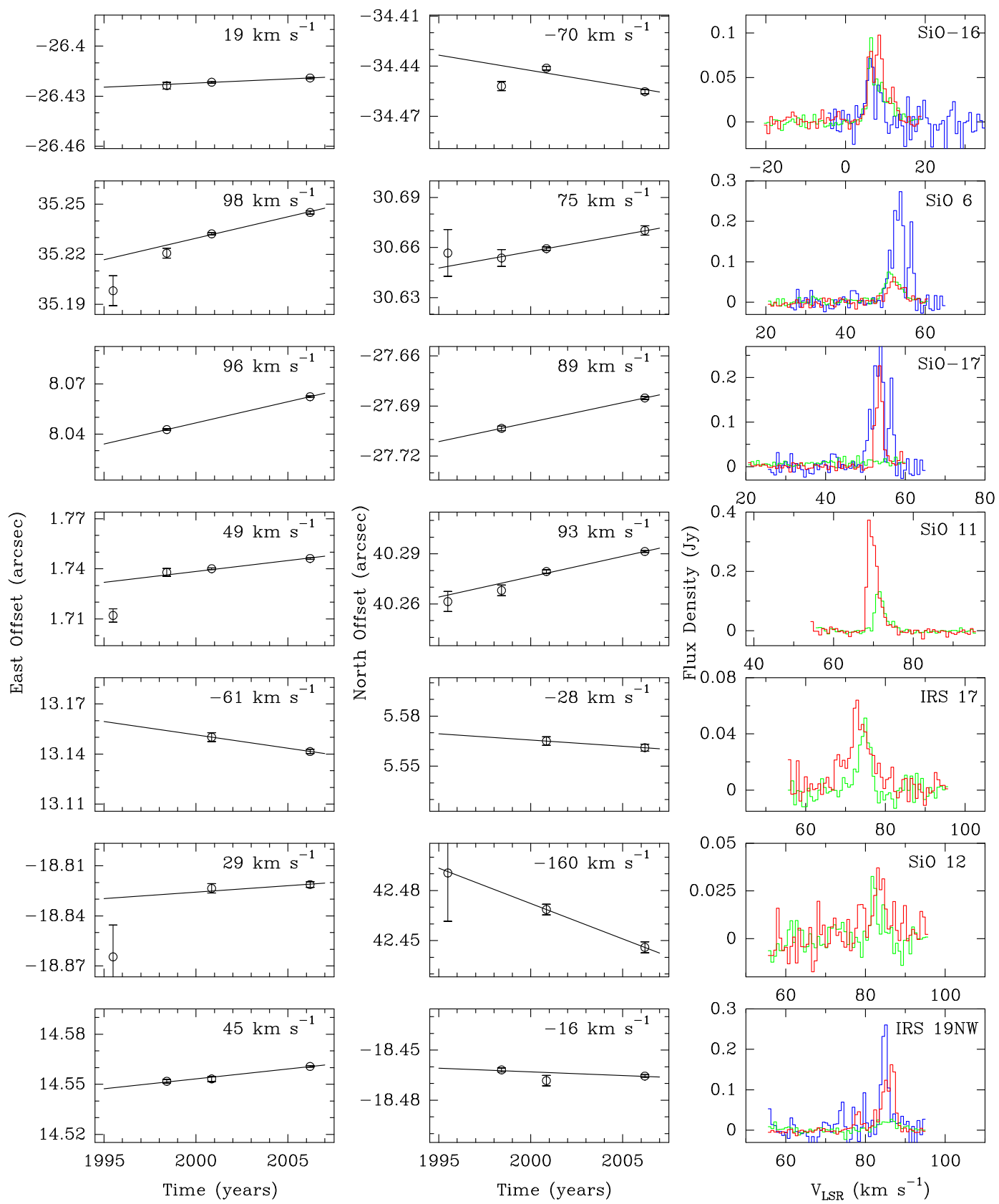

Fig. 3.- Eastward (left panels) and northward (middle panels) position offsets from Sgr A* versus time for the seven $\mathrm{SiO}$ maser stars with positive LSR velocities. Solid lines are variance-weighted best-fit proper motions. The linear speed is indicated in each frame, assuming a distance of $8.0 \mathrm{kpc}$. Spectra from 1998 (blue), 2000 (green) and 2006 (red) observations are also shown (right panels). Star names are indicated in the upper right corner of the right panels. 


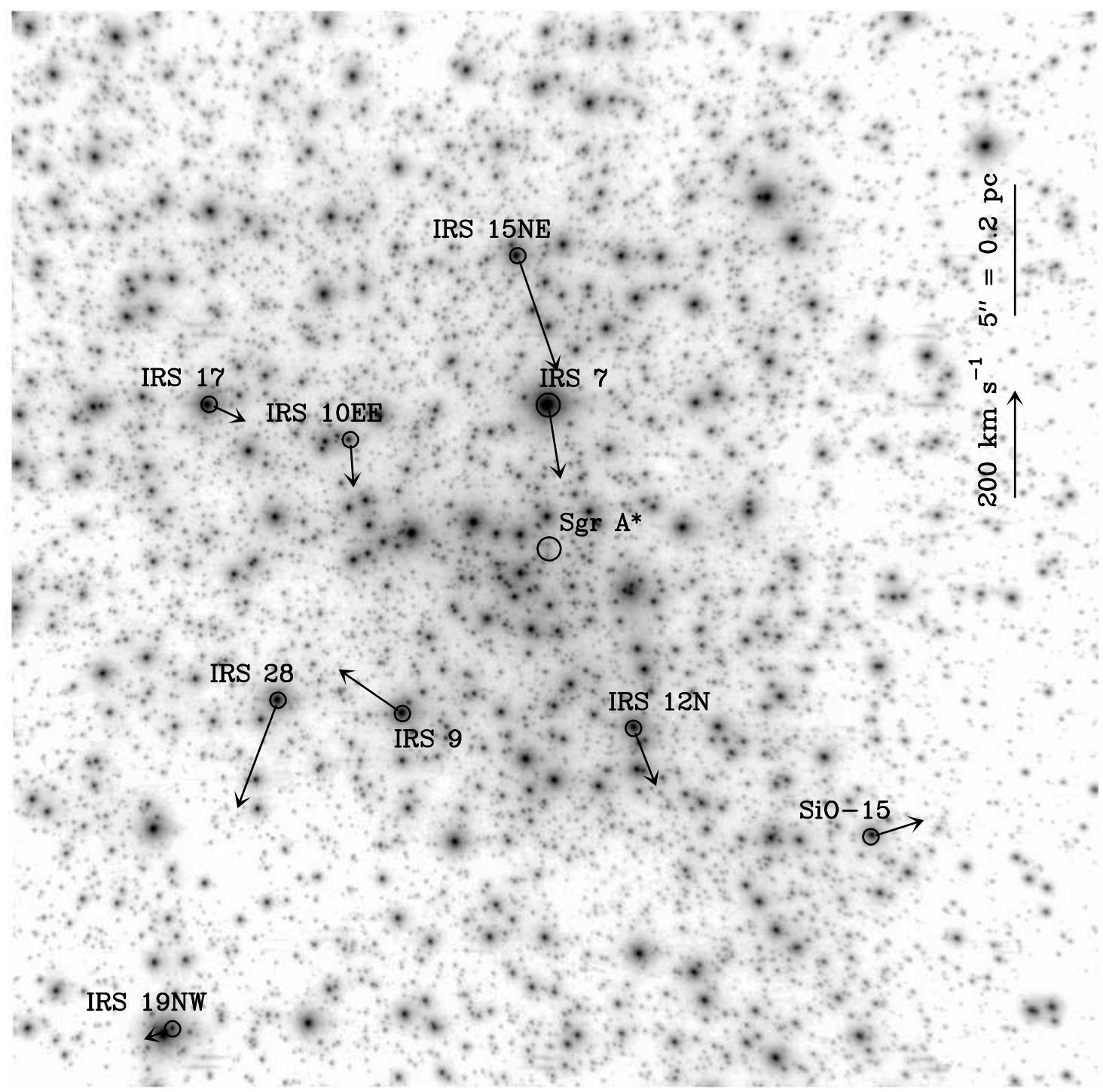

Fig. 4. - Infrared (K-band) image of the central \pm 20 " of the Galactic Center taken in 2005, with east to the left and north to the top. SiO maser stars within this region are circled and their proper motions relative to Sgr A* are indicated with arrows. The vertical bar and arrow at the right of the image indicate the linear and motion scales for $R_{0}=8.0 \mathrm{kpc}$. The location of $\operatorname{Sgr} \mathrm{A}^{*}$ is indicated by the circle at the center of the image. 


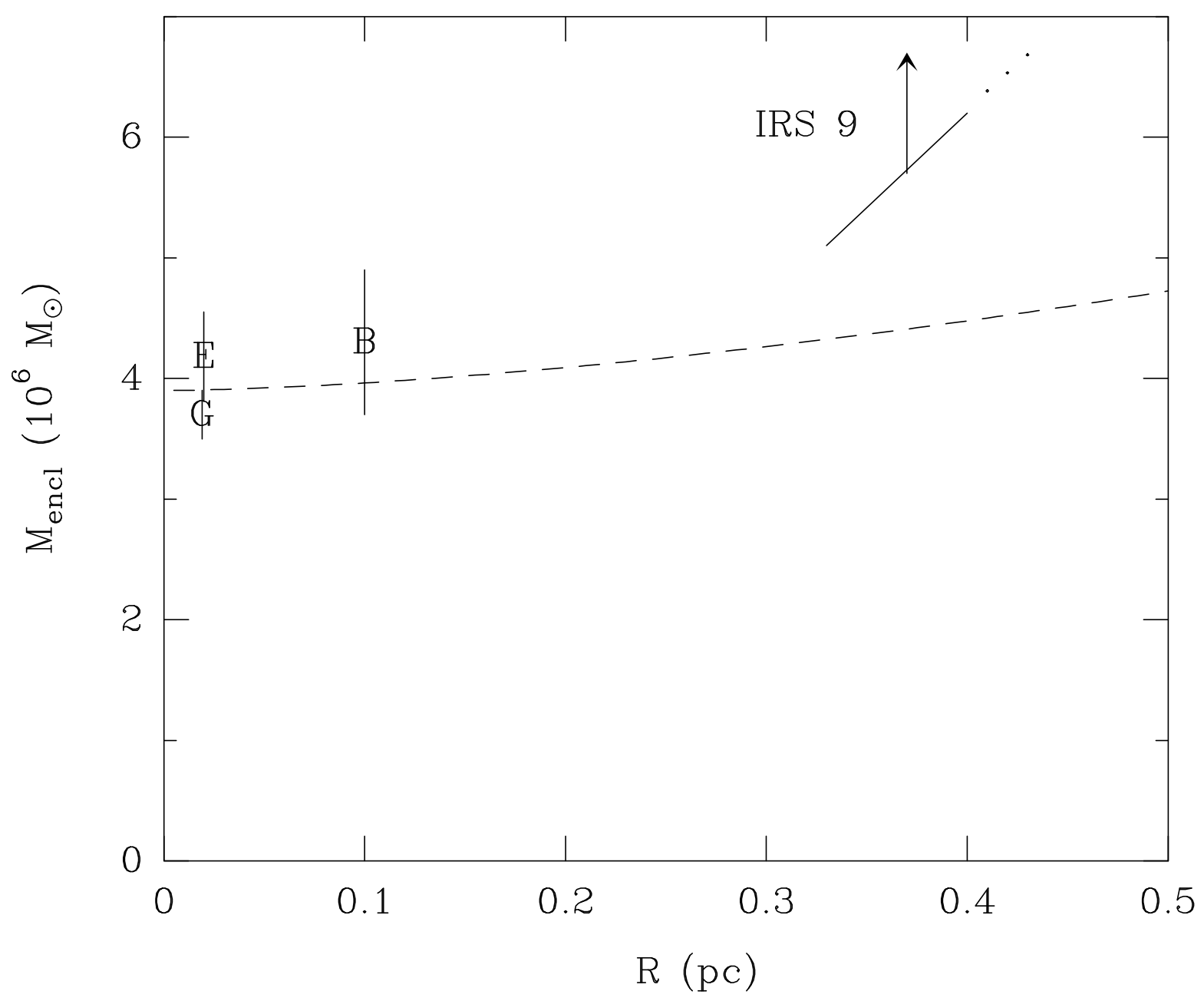

Fig. 5.- Constraints on the enclosed mass as a function of radius $(R)$ from $\operatorname{Sgr} \mathrm{A}^{*}$. The estimates labeled "E" and "G" are from fitting stellar orbits by Eisenhauer et al. (2005) and Ghez et al. (2005), respectively, and the estimate labeled "B" is from a statistical analysis of the "clockwise stellar disk" by Beloborodov et al. (2006). Our lower limit on enclosed mass from the 3-dimensional motion of IRS 9, assuming that it is bound in the region dominated gravitationally by Sgr A* (see $\$ 5.2$ ), is indicated by a sloping line and arrow. The minimum distance of IRS 9 from Sgr A* is its projected distance of $0.33 \mathrm{pc}$. The sloping line corresponds to the mass limit for reasonable values of the unknown line-of-sight distance of IRS 9 from Sgr A*; this increases both the radius and the mass limit linearly. The dashed line indicates the combined contribution of a point mass of $3.9 \times 10^{6} \mathrm{M}_{\odot}$ and a central stellar cusp (Genzel et al. 2003). The uncertainty in the dashed line is dominated by the uncertainty of $\pm 0.2 \times 10^{6} \mathrm{M}_{\odot}$ in the point-mass, with a smaller contribution of perhaps $\pm 0.1 \times 10^{6} \mathrm{M}_{\odot}$ from the central stellar cusp at $R=0.33 \mathrm{pc}$. All values assume $R_{0}=8.0 \mathrm{kpc}$; the discrepancy between the dashed line and the IRS 9 limit cannot be removed for values of $R_{0}<9 \mathrm{kpc}$. 
Table 1. SiO Maser Astrometry

\begin{tabular}{|c|c|c|c|c|c|}
\hline Star & $\begin{array}{c}V_{\mathrm{LSR}} \\
\left(\mathrm{km} \mathrm{s}^{-1}\right)\end{array}$ & $\begin{array}{c}\Delta \Theta_{x} \\
(\operatorname{arcsec})\end{array}$ & $\begin{array}{c}\Delta \Theta_{y} \\
(\operatorname{arcsec})\end{array}$ & $\begin{array}{l}\text { Epoch } \\
(y)\end{array}$ & Telescope \\
\hline IRS $9 \ldots$. & -342 & $\begin{array}{l}5.6515 \pm 0.0048 \\
5.6501 \pm 0.0007 \\
5.6589 \pm 0.0011 \\
5.6742 \pm 0.0004\end{array}$ & $\begin{array}{l}-6.3589 \pm 0.0060 \\
-6.3509 \pm 0.0013 \\
-6.3454 \pm 0.0017 \\
-6.3347 \pm 0.0009\end{array}$ & $\begin{array}{l}1998.39 \\
1998.41 \\
2000.85 \\
2006.20\end{array}$ & $\begin{array}{l}\text { VLA } \\
\text { VLA } \\
\text { VLA } \\
\text { VLA }\end{array}$ \\
\hline IRS $7 \ldots$. & -114 & $\begin{array}{l}0.0403 \pm 0.0080 \\
0.0387 \pm 0.0023 \\
0.0378 \pm 0.0043 \\
0.0342 \pm 0.0016 \\
0.0326 \pm 0.0007\end{array}$ & $\begin{array}{l}5.5829 \pm 0.0130 \\
5.5676 \pm 0.0049 \\
5.5495 \pm 0.0014 \\
5.5414 \pm 0.0030 \\
5.5238 \pm 0.0013\end{array}$ & $\begin{array}{l}1995.49 \\
1998.39 \\
1998.41 \\
2000.85 \\
2006.20\end{array}$ & $\begin{array}{l}\text { VLA } \\
\text { VLA } \\
\text { VLA } \\
\text { VLA } \\
\text { VLA }\end{array}$ \\
\hline $\mathrm{SiO}-14 \ldots .$. & -112 & $\begin{array}{l}-7.6648 \pm 0.0012 \\
-7.6596 \pm 0.0005 \\
-7.6485 \pm 0.0005\end{array}$ & $\begin{array}{l}-28.4528 \pm 0.0020 \\
-28.4530 \pm 0.0008 \\
-28.4586 \pm 0.0009\end{array}$ & $\begin{array}{l}1998.41 \\
2000.85 \\
2006.20\end{array}$ & $\begin{array}{l}\text { VLA } \\
\text { VLA } \\
\text { VLA }\end{array}$ \\
\hline IRS $12 \mathrm{~N} \ldots$. & -63 & $\begin{array}{l}-3.2519 \pm 0.0005 \\
-3.2541 \pm 0.0005 \\
-3.2543 \pm 0.0010 \\
-3.2554 \pm 0.0009 \\
-3.2626 \pm 0.0009\end{array}$ & $\begin{array}{l}-6.8814 \pm 0.0005 \\
-6.8877 \pm 0.0006 \\
-6.8860 \pm 0.0011 \\
-6.8936 \pm 0.0012 \\
-6.9073 \pm 0.0019\end{array}$ & $\begin{array}{l}1996.41 \\
1998.39 \\
1998.41 \\
2000.85 \\
2006.20\end{array}$ & $\begin{array}{l}\text { VLBA } \\
\text { VLA } \\
\text { VLA } \\
\text { VLA } \\
\text { VLA }\end{array}$ \\
\hline IRS $28 \ldots \ldots$ & -55 & $\begin{array}{l}10.4702 \pm 0.0030 \\
10.4693 \pm 0.0010 \\
10.4809 \pm 0.0007\end{array}$ & $\begin{array}{l}-5.7884 \pm 0.0050 \\
-5.7956 \pm 0.0024 \\
-5.8254 \pm 0.0010\end{array}$ & $\begin{array}{l}1998.41 \\
2000.85 \\
2006.20\end{array}$ & $\begin{array}{l}\text { VLA } \\
\text { VLA } \\
\text { VLA }\end{array}$ \\
\hline $\mathrm{SiO}-15 \ldots .$. & -36 & $\begin{array}{l}-12.4253 \pm 0.0023 \\
-12.4385 \pm 0.0012\end{array}$ & $\begin{array}{l}-11.0794 \pm 0.0054 \\
-11.0753 \pm 0.0015\end{array}$ & $\begin{array}{l}2000.85 \\
2006.20\end{array}$ & $\begin{array}{l}\text { VLA } \\
\text { VLA }\end{array}$ \\
\hline IRS 10EE ... & -27 & $\begin{array}{l}7.6821 \pm 0.0030 \\
7.6837 \pm 0.0005 \\
7.6841 \pm 0.0005 \\
7.6837 \pm 0.0005 \\
7.6845 \pm 0.0005 \\
7.6840 \pm 0.0005\end{array}$ & $\begin{array}{l}4.2125 \pm 0.0050 \\
4.2194 \pm 0.0005 \\
4.2146 \pm 0.0009 \\
4.2157 \pm 0.0005 \\
4.2099 \pm 0.0005 \\
4.1990 \pm 0.0005\end{array}$ & $\begin{array}{l}1995.49 \\
1996.41 \\
1998.39 \\
1998.41 \\
2000.85 \\
2006.20\end{array}$ & $\begin{array}{l}\text { VLA } \\
\text { VLBA } \\
\text { VLA } \\
\text { VLA } \\
\text { VLA } \\
\text { VLA }\end{array}$ \\
\hline IRS $15 \mathrm{NE} \ldots$ & -12 & $\begin{array}{l}1.2256 \pm 0.0140 \\
1.2302 \pm 0.0005 \\
1.2249 \pm 0.0017 \\
1.2270 \pm 0.0005 \\
1.2228 \pm 0.0011 \\
1.2112 \pm 0.0005\end{array}$ & $\begin{array}{l}11.3108 \pm 0.0210 \\
11.3315 \pm 0.0005 \\
11.3193 \pm 0.0019 \\
11.3201 \pm 0.0006 \\
11.3024 \pm 0.0025 \\
11.2761 \pm 0.0010\end{array}$ & $\begin{array}{l}1995.49 \\
1996.41 \\
1998.39 \\
1998.41 \\
2000.85 \\
2006.20\end{array}$ & $\begin{array}{l}\text { VLA } \\
\text { VLBA } \\
\text { VLA } \\
\text { VLA } \\
\text { VLA } \\
\text { VLA }\end{array}$ \\
\hline $\mathrm{SiO}-16 \ldots .$. & +8 & $\begin{array}{l}-26.4237 \pm 0.0020 \\
-26.4216 \pm 0.0006\end{array}$ & $\begin{array}{l}-34.4520 \pm 0.0027 \\
-34.4412 \pm 0.0011\end{array}$ & $\begin{array}{l}1998.41 \\
2000.85\end{array}$ & $\begin{array}{l}\text { VLA } \\
\text { VLA }\end{array}$ \\
\hline
\end{tabular}


Table 1-Continued

\begin{tabular}{|c|c|c|c|c|c|}
\hline Star & $\begin{array}{c}V_{\mathrm{LSR}} \\
\left(\mathrm{km} \mathrm{s}^{-1}\right)\end{array}$ & $\begin{array}{c}\Delta \Theta_{x} \\
(\operatorname{arcsec})\end{array}$ & $\begin{array}{c}\Delta \Theta_{y} \\
(\operatorname{arcsec})\end{array}$ & $\begin{array}{l}\text { Epoch } \\
\text { (y) }\end{array}$ & Telescope \\
\hline & & $-26.4191 \pm 0.0006$ & $-34.4553 \pm 0.0013$ & 2006.20 & VLA \\
\hline $\mathrm{SiO}-6 \ldots .$. & +52 & $\begin{array}{l}35.1982 \pm 0.0090 \\
35.2207 \pm 0.0029 \\
35.2323 \pm 0.0006 \\
35.2451 \pm 0.0010\end{array}$ & $\begin{array}{l}30.6567 \pm 0.0140 \\
30.6537 \pm 0.0050 \\
30.6593 \pm 0.0010 \\
30.6702 \pm 0.0028\end{array}$ & $\begin{array}{l}1995.49 \\
1998.39 \\
2000.85 \\
2006.20\end{array}$ & $\begin{array}{l}\text { VLA } \\
\text { VLA } \\
\text { VLA } \\
\text { VLA }\end{array}$ \\
\hline $\mathrm{SiO}-17 \ldots .$. & +53 & $\begin{array}{l}8.0427 \pm 0.0005 \\
8.0624 \pm 0.0005\end{array}$ & $\begin{array}{l}-27.7034 \pm 0.0014 \\
-27.6852 \pm 0.0009\end{array}$ & $\begin{array}{l}1998.41 \\
2006.20\end{array}$ & $\begin{array}{l}\text { VLA } \\
\text { VLA }\end{array}$ \\
\hline $\mathrm{SiO}-11 \ldots$. & +70 & $\begin{array}{l}1.7121 \pm 0.0040 \\
1.7379 \pm 0.0023 \\
1.7401 \pm 0.0005 \\
1.7462 \pm 0.0005\end{array}$ & $\begin{array}{l}40.2614 \pm 0.0060 \\
40.2681 \pm 0.0032 \\
40.2794 \pm 0.0011 \\
40.2914 \pm 0.0006\end{array}$ & $\begin{array}{l}1995.49 \\
1998.39 \\
2000.85 \\
2006.20\end{array}$ & $\begin{array}{l}\text { VLA } \\
\text { VLA } \\
\text { VLA } \\
\text { VLA }\end{array}$ \\
\hline IRS $17 \ldots \ldots$ & +73 & $\begin{array}{l}13.1501 \pm 0.0026 \\
13.1415 \pm 0.0013\end{array}$ & $\begin{array}{l}5.5651 \pm 0.0025 \\
5.5611 \pm 0.0021\end{array}$ & $\begin{array}{l}2000.85 \\
2006.20\end{array}$ & $\begin{array}{l}\text { VLA } \\
\text { VLA }\end{array}$ \\
\hline $\mathrm{SiO}-12 \ldots$. & +82 & $\begin{array}{l}-18.8645 \pm 0.0190 \\
-18.8235 \pm 0.0028 \\
-18.8212 \pm 0.0017\end{array}$ & $\begin{array}{l}42.4905 \pm 0.0290 \\
42.4686 \pm 0.0032 \\
42.4459 \pm 0.0033\end{array}$ & $\begin{array}{l}1995.49 \\
2000.85 \\
2006.20\end{array}$ & $\begin{array}{l}\text { VLA } \\
\text { VLA } \\
\text { VLA }\end{array}$ \\
\hline IRS $19 \mathrm{NW} \ldots .$. & +84 & $\begin{array}{l}14.5518 \pm 0.0011 \\
14.5532 \pm 0.0015 \\
14.5607 \pm 0.0005\end{array}$ & $\begin{array}{l}-18.4619 \pm 0.0012 \\
-18.4683 \pm 0.0031 \\
-18.4656 \pm 0.0009\end{array}$ & $\begin{array}{l}1998.41 \\
2000.85 \\
2006.20\end{array}$ & $\begin{array}{l}\text { VLA } \\
\text { VLA } \\
\text { VLA }\end{array}$ \\
\hline
\end{tabular}

Note. - VLBA $\Delta \Theta_{x}$ and $\Delta \Theta_{y}$ are in the J2000 coordi 
Table 2. SiO Maser Proper Motions

\begin{tabular}{|c|c|c|c|c|c|c|c|}
\hline Star & $\begin{array}{c}V_{\mathrm{LSR}} \\
\left(\mathrm{km} \mathrm{s}^{-1}\right)\end{array}$ & $\begin{array}{c}\Delta \Theta_{x} \\
(\operatorname{arcsec})\end{array}$ & $\begin{array}{c}\Delta \Theta_{y} \\
(\operatorname{arcsec})\end{array}$ & $\begin{array}{c}\mu_{x} \\
\left(\operatorname{mas~}^{-1}\right)\end{array}$ & $\begin{array}{c}\mu_{y} \\
\left(\operatorname{mas}^{-1}\right)\end{array}$ & $\begin{array}{c}\text { Epoch } \\
(y)\end{array}$ & $\begin{array}{l}\text { Number } \\
\text { Epochs }\end{array}$ \\
\hline IRS $9 \ldots \ldots$ & -342 & $+5.6655 \pm 0.0003$ & $-6.3407 \pm 0.0007$ & $+3.06 \pm 0.10$ & $+2.11 \pm 0.19$ & 2003.34 & 3 \\
\hline IRS $7 \ldots \ldots$ & -114 & $+0.0336 \pm 0.0050$ & $+5.5401 \pm 0.0050$ & $-0.58 \pm 0.50$ & $-3.52 \pm 0.54$ & 2004.37 & 4 \\
\hline $\mathrm{SiO}-14 \ldots$. & -112 & $-7.6554 \pm 0.0003$ & $-28.4553 \pm 0.0006$ & $+2.08 \pm 0.12$ & $-0.94 \pm 0.20$ & 2002.89 & 3 \\
\hline IRS $12 \mathrm{~N} \ldots$ & -63 & $-3.2537 \pm 0.0003$ & $-6.8864 \pm 0.0003$ & $-1.06 \pm 0.10$ & $-2.70 \pm 0.17$ & 1998.17 & 4 \\
\hline IRS $28 \ldots .$. & -55 & $+10.4784 \pm 0.0011$ & $-5.8190 \pm 0.0010$ & $+2.00 \pm 0.38$ & $-5.29 \pm 0.42$ & 2005.00 & 3 \\
\hline $\mathrm{SiO}-15 \ldots .$. & -36 & $-12.4372 \pm 0.0011$ & $-11.0757 \pm 0.0015$ & $-2.47 \pm 0.98$ & $+0.77 \pm 2.10$ & 2005.68 & 2 \\
\hline IRS 10EE ... & -27 & $+7.6840 \pm 0.0003$ & $+4.2114 \pm 0.0003$ & $+0.04 \pm 0.07$ & $-2.09 \pm 0.07$ & 2000.24 & 5 \\
\hline IRS $15 \mathrm{NE} \ldots$ & -12 & $+1.2257 \pm 0.0003$ & $+11.3171 \pm 0.0004$ & $-1.96 \pm 0.07$ & $-5.68 \pm 0.12$ & 1998.92 & 5 \\
\hline $\mathrm{SiO}-16 \ldots .$. & +8 & $-26.4207 \pm 0.0004$ & $-34.4478 \pm 0.0043$ & $+0.49 \pm 0.15$ & $-1.84 \pm 1.52$ & 2002.82 & 3 \\
\hline $\mathrm{SiO}-6 \ldots .$. & +52 & $+35.2333 \pm 0.0011$ & $+30.6605 \pm 0.0009$ & $+2.58 \pm 0.43$ & $+1.99 \pm 0.52$ & 2001.43 & 4 \\
\hline $\mathrm{SiO}-17 \ldots .$. & +53 & $+8.0560 \pm 0.0004$ & $-27.6911 \pm 0.0008$ & $+2.53 \pm 0.18$ & $+2.34 \pm 0.42$ & 2003.68 & 2 \\
\hline $\mathrm{SiO}-11 \ldots$. & +70 & $+1.7441 \pm 0.0014$ & $+40.2871 \pm 0.0007$ & $+1.30 \pm 0.46$ & $+2.45 \pm 0.25$ & 2004.38 & 4 \\
\hline IRS $17 \ldots \ldots$ & +73 & $+13.1442 \pm 0.0012$ & $+5.5624 \pm 0.0016$ & $-1.61 \pm 1.08$ & $-0.75 \pm 1.22$ & 2004.49 & 2 \\
\hline $\mathrm{SiO}-12 \ldots$. & +82 & $-18.8227 \pm 0.0030$ & $+42.4559 \pm 0.0023$ & $+0.77 \pm 1.14$ & $-4.24 \pm 0.84$ & 2003.83 & 3 \\
\hline IRS 19NW .. & +84 & $+14.5578 \pm 0.0005$ & $-18.4647 \pm 0.0012$ & $+1.19 \pm 0.14$ & $-0.43 \pm 0.31$ & 2003.79 & 3 \\
\hline
\end{tabular}


Table 3. 3-Dimensional Stellar Motions \& Enclosed Mass Limits

\begin{tabular}{|c|c|c|c|c|c|c|}
\hline Star & $\begin{array}{c}V_{\mathrm{LSR}} \\
\left(\mathrm{km} \mathrm{s}^{-1}\right)\end{array}$ & $\begin{array}{c}V_{x} \\
\left(\mathrm{~km} \mathrm{~s}^{-1}\right)\end{array}$ & $\begin{array}{c}V_{y} \\
\left(\mathrm{~km} \mathrm{~s}^{-1}\right)\end{array}$ & $\begin{array}{c}V_{\text {total }} \\
\left(\mathrm{km} \mathrm{s}^{-1}\right)\end{array}$ & $\begin{array}{c}\mathrm{R}_{\text {proj }} \\
\text { (pc) }\end{array}$ & $\begin{array}{c}\mathrm{M}_{\text {encl }} \\
\left(10^{6} \mathrm{M}_{\odot}\right)\end{array}$ \\
\hline IRS $9 \ldots$ & $-342 \pm 3$ & $116 \pm 4$ & $80 \pm 7$ & $370 \pm 3$ & 0.33 & $>5.1$ \\
\hline IRS $7 \ldots$ & $-114 \pm 3$ & $-22 \pm 19$ & $-133 \pm 20$ & $177 \pm 16$ & 0.21 & $>0.5$ \\
\hline $\mathrm{SiO}-14 \quad \ldots$ & $-112 \pm 3$ & $79 \pm 5$ & $-36 \pm 8$ & $142 \pm 4$ & 1.14 & $>2.4$ \\
\hline IRS $12 \mathrm{~N} \quad \ldots$ & $-63 \pm 3$ & $-40 \pm 4$ & $-102 \pm 6$ & $127 \pm 6$ & 0.30 & $>0.5$ \\
\hline IRS $28 \ldots$ & $-55 \pm 3$ & $76 \pm 14$ & $-201 \pm 16$ & $221 \pm 15$ & 0.46 & $>2.0$ \\
\hline $\mathrm{SiO}-15 \ldots$ & $-36 \pm 3$ & $-94 \pm 37$ & $29 \pm 80$ & $105 \pm 40$ & 0.65 & $>0.0$ \\
\hline IRS $10 \mathrm{EE} \ldots$ & $-27 \pm 3$ & $-5 \pm 3$ & $-82 \pm 3$ & $87 \pm 3$ & 0.34 & $>0.3$ \\
\hline IRS $15 \mathrm{NE} \ldots$ & $-12 \pm 3$ & $-74 \pm 3$ & $-215 \pm 5$ & $228 \pm 4$ & 0.44 & $>2.5$ \\
\hline $\mathrm{SiO}-16 \quad \ldots$ & $8 \pm 3$ & $19 \pm 6$ & $-70 \pm 58$ & $73 \pm 55$ & 1.68 & $>0.0$ \\
\hline $\mathrm{SiO}-6 \quad \ldots$ & $52 \pm 3$ & $98 \pm 16$ & $75 \pm 20$ & $134 \pm 16$ & 1.81 & $>2.2$ \\
\hline $\mathrm{SiO}-17 \quad \ldots$ & $53 \pm 3$ & $96 \pm 3$ & $89 \pm 8$ & $141 \pm 6$ & 1.12 & $>2.2$ \\
\hline $\mathrm{SiO}-11 \quad \ldots$ & $70 \pm 3$ & $49 \pm 17$ & $93 \pm 9$ & $126 \pm 10$ & 1.56 & $>2.1$ \\
\hline IRS $17 \ldots$ & $73 \pm 3$ & $-61 \pm 41$ & $-28 \pm 46$ & $99 \pm 29$ & 0.55 & $>0.1$ \\
\hline $\mathrm{SiO}-12 \quad \ldots$ & $82 \pm 3$ & $29 \pm 43$ & $-160 \pm 32$ & $183 \pm 29$ & 1.80 & $>3.3$ \\
\hline IRS $19 \mathrm{NW} \ldots$ & $84 \pm 3$ & $45 \pm 5$ & $-16 \pm 12$ & $97 \pm 4$ & 0.91 & $>0.8$ \\
\hline
\end{tabular}

Note. $-V_{x}$ and total speed of the s assume a distance $t$ 
Table 4. Radio-Infrared Proper Motion Alignment

\begin{tabular}{|c|c|c|c|c|c|c|}
\hline Star & $\begin{array}{c}\mu_{x}^{\text {Radio }} \\
\left(\text { mas y }^{-1}\right)\end{array}$ & $\begin{array}{c}\mu_{y}^{\text {Radio }} \\
\left(\text { mas y }^{-1}\right)\end{array}$ & $\begin{array}{c}\mu_{x}^{I R} \\
\left(\operatorname{mas~y}^{-1}\right)\end{array}$ & $\begin{array}{c}\mu_{y}^{I R} \\
\left(\operatorname{mas~y}^{-1}\right)\end{array}$ & $\begin{array}{c}\mu_{x}^{D i f} \\
\left(\operatorname{mas~}^{-1}\right)\end{array}$ & $\begin{array}{c}\mu_{y}^{D i f} \\
\left(\operatorname{mas~}^{-1}\right)\end{array}$ \\
\hline IRS9 & $3.06 \pm 0.10$ & $2.11 \pm 0.19$ & $4.44 \pm 0.55$ & $1.10 \pm 0.58$ & $1.38 \pm 0.56$ & $-1.01 \pm 0.61$ \\
\hline IRS7 $\ldots$ & $-0.58 \pm 0.50$ & $-3.52 \pm 0.54$ & $1.86 \pm 1.18$ & $-7.33 \pm 0.72$ & $2.44 \pm 1.28$ & $-3.81 \pm 0.90$ \\
\hline IRS12N $\ldots$ & $-1.06 \pm 0.10$ & $-2.70 \pm 0.17$ & $-0.77 \pm 0.66$ & $-3.39 \pm 0.39$ & $0.29 \pm 0.67$ & $-0.69 \pm 0.43$ \\
\hline IRS28 $\ldots$ & $2.00 \pm 0.38$ & $-5.29 \pm 0.42$ & $2.27 \pm 0.35$ & $-5.85 \pm 0.31$ & $0.27 \pm 0.52$ & $-0.56 \pm 0.52$ \\
\hline $\mathrm{SiO}-15 \quad \ldots$ & $-2.47 \pm 0.98$ & $0.77 \pm 2.10$ & $-2.18 \pm 0.51$ & $-0.56 \pm 0.12$ & $0.29 \pm 1.10$ & $-1.33 \pm 2.10$ \\
\hline IRS10EE $\ldots$ & $0.04 \pm 0.08$ & $-2.09 \pm 0.07$ & $0.73 \pm 0.23$ & $-1.92 \pm 0.27$ & $0.69 \pm 0.24$ & $0.17 \pm 0.28$ \\
\hline IRS15NE $\ldots$ & $-1.96 \pm 0.07$ & $-5.68 \pm 0.12$ & $-2.40 \pm 0.48$ & $-6.29 \pm 0.35$ & $-0.44 \pm 0.49$ & $-0.61 \pm 0.37$ \\
\hline IRS17 $\ldots$ & $-1.61 \pm 1.08$ & $-0.75 \pm 1.22$ & $0.20 \pm 0.59$ & $-1.67 \pm 0.62$ & $1.81 \pm 1.23$ & $-0.92 \pm 1.37$ \\
\hline IRS19NW $\ldots$ & $1.19 \pm 0.14$ & $-0.43 \pm 0.31$ & $-0.60 \pm 3.08$ & $-0.54 \pm 3.47$ & $-1.79 \pm 3.08$ & $-0.11 \pm 3.48$ \\
\hline
\end{tabular}

Note. $-\mu_{x}$ and (infrared minus rad motions are relative 